\title{
3D MRI for Quantitative Analysis of Quadrant Percent Breast Density: Correlation with Quadrant Location of Breast Cancer
}

\author{
Jeon-Hor Chen ${ }^{1,2}$, Fuyi Liao ${ }^{1}$, Yang Zhang ${ }^{1}$, Yifan $\mathrm{Li}^{1}$, Chia-Ju Chang ${ }^{3}$, Chen-Pin Chou ${ }^{4}$, \\ Tsung-Lung Yang ${ }^{4}$, and Min-Ying Su ${ }^{1}$ \\ ${ }^{1}$ Center for Functional Onco-Imaging, Department of Radiological Sciences, University of \\ California, Irvine, CA, United States \\ 2Department of Radiology, E-Da Hospital and I-Shou University, Kaohsiung, Taiwan \\ ${ }^{3}$ Department of Radiological Technology, China Medical University, Taichung, Taiwan \\ ${ }^{4}$ Department of Radiology, Kaohsiung Veterans General Hospital, Kaohsiung, Taiwan
}

\section{Abstract}

Rationale and Objectives-Breast cancer occurs more frequently in the upper outer (UO) quadrant, but whether this higher cancer incidence is related to the greater amount of dense tissue is not known. MRI acquires 3-dimensional volumetric images and is the most suitable among all breast imaging modalities for regional quantification of density. This study applied an MRI-based method to measure quadrant percent density (QPD), and evaluated its association with the quadrant location of the developed breast cancer.

\begin{abstract}
Materials and Methods-A total of 126 cases with pathologically confirmed breast cancer were reviewed. Only women who had unilateral breast cancer located in a clear quadrant were selected for analysis. A total of 84 women, including 47 Asian women and 37 Western women, were included. An established computer-aided method was used to segment the diseased breast and the contralateral normal breast, and to separate the dense and fatty tissues. Then a breast was further separated into four quadrants by using the nipple and the centroid as anatomic landmarks. The tumor was segmented by using a computer-aided method to determine its quadrant location. The distribution of cancer quadrant location, quadrant with the highest QPD, and the proportion of cancers occurring in the highest QPD were analyzed.
\end{abstract}

Results-The highest incidence of cancer occurred in the upper outer (UO) quadrant (36/84, 42.9\%). The highest QPD was also noted most frequently in the UO quadrant $(31 / 84,36.9 \%)$. When correlating the highest QPD with the quadrant location of breast cancer, only 17 women $(17 / 84,20.2 \%)$ had breast cancer occurring in the quadrant with the highest QPD.

\footnotetext{
*Corresponding Author: Jeon-Hor Chen, M.D., John Tu and Thomas Yuen Center for Functional Onco-Imaging, 164, Irvine Hall, University of California, Irvine, CA 92697-5020, USA, Tel: (949) 824-9327, Fax: (949) 824-3481, jeonhc@uci.edu.

Publisher's Disclaimer: This is a PDF file of an unedited manuscript that has been accepted for publication. As a service to our customers we are providing this early version of the manuscript. The manuscript will undergo copyediting, typesetting, and review of the resulting proof before it is published in its final form. Please note that during the production process errors may be discovered which could affect the content, and all legal disclaimers that apply to the journal pertain.
} 
Conclusions-The results showed that the development of breast cancer in specific quadrant could not be explained by the density in that quadrant, and further studies are needed to find the biological reasons accounting for the higher breast cancer incidence in the UO quadrant.

\section{Introduction}

Mammographic density (MD) is an independent risk factor for development of breast cancer (1-2). The biological basis for the association between increased breast cancer risk and higher MD is not fully understood. Studies of mammographically dense tissues suggest that density may represent increased epithelial cellular concentration, stromal fibrosis, and epithelial hyperplasia (3). A fundamental question that has yet to be answered is whether cancers tend to arise in mammographically dense tissue. Among several studies exploring the question, two studies showed that tumors occur overwhelmingly in the mammographically dense areas, suggesting that some aspect of glandular/stromal tissue comprising the dense tissue directly influences the carcinogenic process (4-5). Another study, however, found that after accounting for overall density, the regional density was not a significant risk factor for subsequently developed cancer (6).

Many studies have shown the quadrant disparity of cancer risk and noted that the upper outer (UO) quadrant was the most frequent site of carcinoma (7-9). A study consisting of 746 consecutive breast core biopsies found $62 \%$ of 349 malignant lesions (95\% confidence interval 57-67\%) occurred in the UO quadrant (8). An adequate explanation for this asymmetric occurrence of breast cancer within the breast has never been established. Is the disparity of breast cancer in different quadrants related to the amount of breast density? As the first step to answer this question, it is necessary to develop a reliable quantification method to measure quadrant breast density. Most published studies analyzed the cancer risk related to whole breast density, which did not consider the spatial variation of the dense tissue in the breast.

Three studies analyzed the quadrant density based on mammography before (4-6). With mammography, the craniocaudal (CC) and mediolateral oblique (MLO) image could be divided into two regions; i.e., the $\mathrm{CC}$ image was divided into a lateral and medial part (CC-L and CC-M); and the MLO image was divided into a superior and inferior part (MLO-S and MLO-I) $(4,6)$. The quadrant breast density was estimated as the average of the density assessments of two mammographic areas. For example, the UO quadrant density is the average density of the CC-Lateral and MLO-Superior areas. Since mammography acquires 2-dimensional projection images, this method could only provide a reasonable estimate, not a true measure, of density in four quadrants. Furthermore, traditional mammography is not able to provide quantitative volumetric measures.

Breast MRI acquires 3-dimensional volumetric images that show a good contrast between fibroglandular (dense) and fatty tissues, and well-developed segmentation methods capable of measuring breast volume and fibroglandular tissue volume quantitatively have been reported $(10,11)$. In this work we applied a MR-based quadrant separation method to measure quadrant percent density (QPD), and evaluated its association with the quadrant location of the developed breast cancer (12). Bilateral breasts in a healthy woman can be 
assumed to be symmetrical (13-15). Since the density in the diseased breast has been altered with the presence of tumor, we analyzed the QPD in the normal contralateral breast to simulate the density in the diseased breast before the cancer occurred.

\section{Materials and Methods}

\section{Subjects}

This study was approved by the institutional review board and complied with the Health Insurance Portability and Accountability Act. A total of 126 cases with pathologically confirmed breast cancer were reviewed. Women with bilateral breast cancer and women with unilateral breast cancer, but the cancer occupied more than two quadrants or located in the subareolar area were excluded from the study. Only those women who had unilateral breast cancer located in a clear dominant quadrant location were selected for analysis. In total, 84 women (mean age 49, range 28-70) were included. Thirty-seven of these subjects were western women from one institution. These women had advanced invasive breast cancer and received baseline and several follow-up breast MRI studies following the neoadjuvant chemotherapy. In this study, only the baseline breast MR images were used for density analysis. The tumor size of these thirty-seven women measured in baseline breast MRI ranged from $1.7 \mathrm{~cm}$ to $8.8 \mathrm{~cm}$ (mean $\pm \mathrm{STD}=4.1 \pm 1.8 \mathrm{~cm})$. Forty-seven subjects were Asian women from a diagnostic setting in another institution. These subjects received diagnostic breast MRI studies due to various clinical reasons, such as palpable breast lumps or suspicious breast lesions detected by mammography or breast ultrasonographic studies, and were found to have unilateral breast cancer. Of these forty-seven women, twenty-nine women were found to have invasive cancer and eighteen women had ductal carcinoma in situ (DCIS). The tumor size of these forty-seven women measured in the preoperative diagnostic breast MRI ranged from $0.3 \mathrm{~cm}$ to $4.2 \mathrm{~cm}$ (mean $\pm \mathrm{STD}=1.7 \pm 1.0 \mathrm{~cm})$. All subjects gave written informed consent to participate in the MRI study.

\section{MR Imaging Acquisition}

In this study only the non-contrast T1-weighted (T1W) images without fat suppression were used for measurement of breast density. For the western women, the MR images were acquired at a 3.0-tesla MR scanner (Philips Achieva, Philips Medical Systems, Best, Netherlands) with a dedicated, sensitivity encoding (SENSE)-enabled, bilateral 4-channel breast coil. The pre-contrast nonfat-suppressed T1W imaging sequence used for the density analysis was $2 \mathrm{D}$ turbo spin-echo, with $\mathrm{TR}=800 \mathrm{~ms}, \mathrm{TE}=8.6 \mathrm{~ms}$, flip angle $=90^{\circ}$, matrix size $=480 x 480$, field of view $(F O V)=31-38 \mathrm{~cm}$, and slice thickness $=2 \mathrm{~mm}$.

The Asian subjects were examined by using a 3.0-tesla MR scanner (Magneton Skyra, Siemens Medical Solutions, Erlangen, Germany), with a 16-channel Sentinelle breast coil. The pre-contrast nonfat-suppressed T1WI imaging sequence used for the density analysis was three-dimensional fast low angle shot (3D- FLASH) sequence, with TR/TE $=4.36 / 1.58$ $\mathrm{ms}$, flip angle $=10$ degrees, number of signal average $=1$, matrix size $=384 \times 288, \mathrm{FOV}=30$ $\mathrm{cm}$, and slice thickness $=1.0 \mathrm{~mm}$. 


\section{Breast and Fibroglandular Tissue Segmentation}

The MR density measurement was done by using an established template-based automatic segmentation method (11). With the method, the chest body region on a middle slice was used as the template. Within the chest template, three body landmarks (thoracic spine and bilateral boundary of the pectoralis muscle) were identified for performing the initial Vshape cut to determine the posterior lateral boundary of the breast. The chest template was mapped to each subject's image space to obtain a subject-specific chest model for exclusion. The chest and muscle boundaries determined on the middle slice were used as the reference for the segmentation of adjacent slices, and the process continued until all 3D slices were segmented. The segmentation of fibroglandular tissue and fatty tissue used nonparametric nonuniformity normalization (N3) + Fuzzy C Means (FCM) algorithm (16). The segmented breast and fibroglandular tissue from all slices were used to calculate the breast volume (BV) and fibroglandular tissue volume (FV), and then the percent density (PD) as FV/BV x $100 \%$. Detailed procedures for the density quantification were reported before (11).

\section{Quadrant Breast Density Assessment}

The quadrant separation was done by using a recently reported method (17). The nipple and the centroid of the segmented breast was used as anatomic landmarks to divide the breast into four quadrants: upper-outer (UO), upper-inner (UI), lower-outer (LO), and lower-inner (LI), by one horizontal plane and one vertical plane intersecting along the aligning nipplecentriod line (Figure 1). Figure 2 illustrates how the breast tissue and fibroglandular tissue are divided into four quadrants. After the four quadrants were separated, the breast volume and fibroglandular tissue volume were measured to calculate the quadrant percent density (QPD). The detailed methods for the quantification of QPD were reported before (17).

\section{Determination of Tumor Quadrant Location}

The quadrant location of the breast cancer in the diseased breast was determined by using a computer-aided method based on the DCE-MRI sequence. The tumor was segmented on the contrast enhancement maps obtained at 2 min after contrast injection, as reported before (18). The operator placed a rectangle box to cover the whole lesion on the maximum intensity projection (MIP), and then the outline of the tumor was obtained by FCM algorithm based on signal intensity. Scattered voxels not connecting to the tumor were removed, and then the hole-filling algorithm was applied to outline the whole tumor. An example is shown in Figure 3. The tumor volume was measured. The separation of the diseased breast into 4 quadrants was done by using the same methods described above for the contralateral normal breast. After the breast quadrants and the tumor were segmented, the location of tumor could be determined. If more than $50 \%$ of the tumor was in a specific quadrant, that quadrant was determined as the dominant quadrant. In this study only cases with a clear dominant quadrant for the occurred tumor were analyzed.

\section{Statistics}

Mean breast volume, fibroglandular tissue volume, and percent density in the four quadrants were calculated. For each case, the quadrant of tumor location and the quadrant with the 
highest QPD were determined for descriptive analysis. The proportion of cases whose tumor occurring in the quadrant with the highest QPD was calculated.

\section{Results}

\section{Quadrant BV, FV, and PD}

Table 1 shows the mean and standard deviation of the breast volume (BV), fibroglandular tissue volume (FV), and percent density (PD) in the four quadrants in Asian and Western women. It was noted that the mean breast volume was the highest in the UO quadrant for both Western and Asian women (breast volume: $308.8 \mathrm{~cm}^{3}$ and $201.8 \mathrm{~cm}^{3}$ respectively). The mean fibroglandular tissue volume was the highest in the UO for the Western women $\left(44.2 \mathrm{~cm}^{3}\right)$ and the highest in the LO for the Asian women $\left(33.1 \mathrm{~cm}^{3}\right)$. The mean QPD for Western women was highest in the UO quadrant (14.4\%) and the highest in the LO quadrant (19.6\%) for Asian women.

\section{Quadrant Location of Tumor and Highest QPD}

Table 2 shows the distribution of breast cancer in different quadrants. The most frequent tumor site was the UO quadrant $(36 / 84,42.9 \%)$, followed by the UI quadrant (20/84, 23.8\%), the same for both Asian and Western women. Table 3 shows the distribution of the highest QPD, and cancers occurring in the highest QPD quadrant. The UO quadrant was the most likely to have the highest QPD $(31 / 84,36.9 \%)$ followed by the LO quadrant $(27 / 84$, $32.1 \%)$. The LI quadrant was the least likely to have the highest QPD $(11 / 84,13.1 \%)$. Overall, only 17 women $(17 / 84,20.2 \%)$ had breast cancer occurring in the quadrant with the highest QPD, with 11 in the UO quadrant. Three examples are shown. Figure 4 shows a 65 y/o woman with a $2.5 \mathrm{~cm}$ invasive cancer in the UO quadrant of the left breast. Figure 5 shows a $56 \mathrm{y} / \mathrm{o}$ woman with a $1.3 \mathrm{~cm}$ invasive cancer in the UI quadrant of the left breast. Figure 6 shows a 38 y/o woman with a $2.9 \mathrm{~cm}$ invasive cancer in the LO quadrant of the right breast.

\section{Discussion}

Although MD is associated with breast cancer risk, it is not known whether MD is directly related to cancer occurrence, i.e., tumors arising within the dense tissue (2). Clarification of whether MD is directly related to risk will enhance our understanding of the pathogenesis of breast cancer and will provide information on the value of using localized MD as a predictor of subsequent tumor location, possibly opening up new avenues for the clinical management of high-risk women (2). The density assessment using MD is, however, fundamentally limited by the fact that mammography is a $2 \mathrm{D}$ projection imaging method and natural distortions between breasts are likely to occur during the course of breast compression used in mammography. As such, the density measures can be confounded by the nature of the imaging procedure itself (14). Breast MRI allows a 3D quantitative measurement of breast volume and fibroglandular tissue volume without suffering from the problem of tissue overlapping. The goal of this study is to measure QPD in the normal breast and evaluate its association with the tumor location in the diseased breast. 
The quantitative analysis of QPD required two computer-aided procedures: first to segment the whole breast and the dense tissue, and second to separate a breast into 4 quadrants. The 3D MR-based density quantification method was done by using a well-developed automatic segmentation algorithm to measure $\mathrm{BV}, \mathrm{FV}$, and PD (11). Then within the segmented breast, the nipple and the centroid was used as references to divide a breast into 4 quadrants for measurement of QPD (16). In most women, the bilateral breasts were in general symmetrical (13-15). In this study, all the 84 patients included had unilateral breast cancer. The tumor size ranged from $0.3 \mathrm{~cm}$ to $8.8 \mathrm{~cm}$. With tumor in one breast, it thus was not possible to evaluate the breast symmetry. However, analysis based on a separate dataset of mammograms in 67 healthy women (unpublished data) we noted that the right-and-left symmetry of percent breast density is pretty high $(r=0.84)$. Despite of the high symmetry, the assessment of symmetry in mammography is potentially limited by the fact that natural distortions between breasts are likely to occur during the course of breast compression routinely used in mammography. As such, symmetry measures can be confounded by the nature of the imaging procedure itself (19). In our recently published results using 3D MRI in the study of breast density in 58 normal women, 47 pre-menopausal and 11 postmenopausal women (17), we found that bilateral breasts in women without cancer are highly symmetrical ( $\mathrm{r}=0.97$ for breast volume, $\mathrm{r}=0.97$ for fibroglandular tissue volume, and $\mathrm{r}=0.98$ for percent breast density). Another study using MRI showed small differences in the bilateral breast tissue composition, i.e. fat and water content, in young women and adults (20).

A study to investigate the spatial distribution of density within the breast using 493 mammographic images from a sample of 165 premenopausal women showed that the degree of the spatial clustering of density was similar between a woman's two breasts, and did not change with aging (13). Therefore, in this study we analyzed the contralateral normal breast to simulate the density in the diseased breast without the presence of the tumor. Two different cohorts, Asian women from diagnostic MRI setting and Western women from more advanced neoadjuvant chemotherapy setting, were studied.

The results showed that breast cancer was the most likely to occur in the UO quadrant in the both cohorts. The finding was consistent with most of the literature reports in studies of the western women (7-9), eastern women (21), and Asian women (22,23). A study of Taiwanese women (23) showed that more than half $(52.3 \%)$ of the primary breast tumors occurred in the UO quadrant. In the MR study the woman was examined in a prone position, and the separation of 4 quadrants in a different breast shape will be different from the traditional assessment while the woman was in a standing position for mammography or a spine position for ultrasound. Our frequency of UO tumor in this MR study was lower than the reports from mammographic studies $(7-9,21-23)$. The UO quadrant is also the most frequent location for benign lesions including fibroadenoma and breast cysts (24) and phyllodes tumor (25). The reasons why breast cancer grows more frequently in the UO quadrant are not clear. The results from a study (8) noted that the high proportion of UO quadrant carcinomas of the breasts is a reflection of the greater amount of breast tissue in this quadrant. In that study, the numbers of core biopsies reported as normal, benign and malignant in each quadrant were used as measures of the frequency of normal tissue, benign disease and malignant disease respectively in the different quadrants. It was noted that the 
proportion of core biopsies from the UO quadrant reported as normal (67\%), benign (57\%) or malignant $(62 \%)$ were similar. However, this study did not measure the mammographic density; thus did not provide sufficient evidence to support the relationship with density. The results from another study (9) did not support that the higher frequency of UO quadrant cancer was due solely to a greater amount of target epithelial tissue in that region. It was postulated that the increasing use of cosmetics applied to the adjacent underarm and upper breast area might account for the findings. The underarm cosmetics are known to contain both DNA-damaging chemicals and chemicals which can mimic estrogen action (26), and greater use of these cosmetics can reduce the age of breast cancer diagnosis (27).

Higher MD has, histologically, a greater cellular concentration and/or proliferation of the stroma or epithelium (3). It was thus postulated that areas of increased density may be more susceptible to the initiation and promotion of breast cancers than areas of lower densities (6). To clarify the question, several mammographic studies $(4,6)$ had been conducted to correlate quadrant breast density with the occurrence of breast cancer but the findings were inconsistent. A study investigated whether tumors arise specifically within dense tissue by generating a virtual $1 \mathrm{~cm}$ square grid overlaid on a mammogram and estimating the odds of a tumor arising in a square in relation to its pre-diagnostic square-specific MD (5). The results showed that tumors arise predominantly within the radiodense breast tissue, but this analysis method could not explain the relationship between density and the high cancer incidence in the UO quadrant. Overall, two-dimensional (2D) mammographic method cannot truly measure volumetric regional density. Our study based on the analysis of 3D MR images noted that only $20.2 \%$ of women had breast cancer occurring in the breast quadrant with the highest QPD. The results agreed with the mammographic study (6) that quadrant breast density was not a significant risk factor for the subsequent development of breast cancer, and that a greater amount of breast tissue in a specific quadrant cannot solely explain the high breast cancer incidence in the UO quadrant (9). A more recent study of genomic patterns of loss of heterozygosity and allelic imbalance in breast quadrants from 21 breast cancer patients showed increased levels of genomic instability in the outer breast quadrants, suggesting that increased levels of breast cancer in the UO quadrant might result from the development of genetic alterations in that region of the breast rather than from only a greater tissue volume (28).

In our study we noted that among the four breast quadrants, the UO quadrant had the highest mean breast volume in both Asian and Western women. But Asian women tended to have the highest mean fibroglandular tissue volume in the LO quadrant, different from the Western women noted in the UO quadrant. This was a finding never been reported before, and large series of studies are needed before any conclusion can be drawn. Other limitations included the small subject number and the two patient groups came from different setting, one from neoadjuvant chemotherapy study and the other from screening and diagnostic study.

In conclusion, we used a recently developed method to quantify the QPD based on MR images, and to investigate whether the PD in a specific breast quadrant was associated with occurrence of breast cancer. We found a higher proportion of women with breast cancer in the UO quadrant, true for both Asian and western women. The UO quadrant was the most 
likely to have the highest QPD among the four quadrants in the western women but not in the Asian women. Only $20 \%$ of women had breast cancer growing in the quadrant with the highest QPD. The results suggested that fibroglandular tissue quantity and density did not explain the asymmetry of tumor distribution among quadrants in women undergoing neoadjuvant chemotherapy, and in women with cancer detected during routine diagnostic breast MRI studies. Therefore, the larger amount of breast tissue alone in a specific quadrant cannot fully explain the disproportional occurrence of breast cancer in different quadrants.

\section{Acknowledgments}

This work was supported in part by NIH/NCI Grant No. R01 CA127927, R21 CA170955, and R03 CA136071.

\section{References}

1. Boyd NF, Guo H, Martin LJ, et al. Mammographic density and the risk and detection of breast cancer. N Engl J Med. 2007; 356(3):227-236. [PubMed: 17229950]

2. Vachon CM, Brandt KR, Ghosh K, et al. Mammographic breast density as a general marker of breast cancer risk. Cancer Epidemiol Biomarkers Prev. 2007; 16(1):43-49. [PubMed: 17220330]

3. Britt K, Ingman W, Huo C, Chew G, Thompson E. The pathobiology of mammographic density. J Cancer Biol Res. 2014; 2(1):1021.

4. Ursin G, Hovanessian-Larsen L, Parisky YR, Pike MC, Wu AH. Greatly increased occurrence of breast cancers in areas of mammographically dense tissue. Breast Cancer Res. 2005; 7(5):R605608. [PubMed: 16168104]

5. Pinto Pereira SM, McCormack VA, Hipwell JH, et al. Localized fibroglandular tissue as a predictor of future tumor location within the breast. Cancer Epidemiol Biomarkers Prev. 2011 Aug; 20(8): 1718-1725. [PubMed: 21693627]

6. Vachon CM, Brandt KR, Ghosh K, et al. Mammographic breast density as a general marker of breast cancer risk. Cancer Epidemiol Biomarkers Prev. 2007 Jan; 16(1):43-49. [PubMed: 17220330]

7. Blumgart EI, Uren RF, Nielsen PM, Nash MP, Reynolds HM. Lymphatic drainage and tumour prevalence in the breast: a statistical analysis of symmetry, gender and node field independence. $\mathrm{J}$ Anat. 2011 Jun; 218(6):652-659. [PubMed: 21453408]

8. Lee AH. Why is carcinoma of the breast more frequent in the upper outer quadrant? A case series based on needle core biopsy diagnoses. Breast. 2005 Apr; 14(2):151-152. [PubMed: 15767185]

9. Darbre PD. Recorded quadrant incidence of female breast cancer in Great Britain suggests a disproportionate increase in the upper outer quadrant of the breast. Anticancer Res. 2005 May-Jun; 25(3c):2543-2550. [PubMed: 16080490]

10. Nie K, Chen JH, Chan S, et al. Development of a quantitative method for analysis of breast density based on 3-dimensional breast MRI. Medical Physics. 2008; 35(12):5253-5262.

11. Lin M, Chen JH, Wang X, Chan S, Chen S, Su MY. Template-based automatic breast segmentation on MRI by excluding the chest region. Med Phys. 2013 Dec.40(12):122301. [PubMed: 24320532]

12. Chen JH, Li Y, Su MY. 3D MRI for quantitative analysis of quadrant percent breast density (QPD): correlation with quadrant location of breast cancer. Annal Onco. 2015; 26(suppl 3):27-28.

13. Pinto Pereira SM, McCormack VA, Moss SM, dos Santos Silva I. The spatial distribution of radiodense breast tissue: a longitudinal study. Breast Cancer Res. 2009; 11(3):R33. [PubMed: 19490643]

14. Alterson R, Plewes DB. Bilateral symmetry analysis of breast MRI. Phys Med Biol. 2003; 48:3431-3443. [PubMed: 14620067]

15. Stines J, Tristant H. The normal breast and its variations in mammography. Eur J Radiol. 2005; 54:26-36. [PubMed: 15797291]

16. Lin M, Chan S, Chen JH, et al. A new bias field correction method combining N3 and FCM for improved segmentation of breast density on MRI. Med Phys. 2011; 38:5-14. [PubMed: 21361169] 
17. Fwu PT, Chen JH, Li Y, Chan S, Su MY. Quantification of Regional Breast Density in Four Quadrants Using 3D MRI-A Pilot Study. Transl Oncol. 2015 Aug; 8(4):250-257. [PubMed: 26310370]

18. Newell D, Nie K, Chen JH, et al. Selection of diagnostic features on breast MRI to differentiate between malignant and benign lesions using computer-aided diagnosis: differences in lesions presenting as mass and non-mass-like enhancement. Eur Radiol. 2010 Apr; 20(4):771-781. [PubMed: 19789878]

19. Alterson R, Plewes DB. Bilateral symmetry analysis of breast MRI. Phys Med Biol. 2003; 48:3431-3443. [PubMed: 14620067]

20. Hennessey S, Huszti E, Gunasekura A, et al. Bilateral symmetry of breast tissue composition by magnetic resonance in young women and adults. Cancer Causes Control. 2014 Apr; 25(4):491497. [PubMed: 24477331]

21. Hussain MA, Sawkat A, Tyagi SP, Reza H. Incidence of cancer breast at Aligarh. J Indian Med Assoc. 1994; 92:296-297. [PubMed: 7814903]

22. Cheung TT, Suen DT, Kwong A. Is sentinel lymph node biopsy after neoadjuvant chemotherapy feasible in Chinese patients with invasive breast cancers? ANZ J Surg. 2009 Oct; 79(10):719-723. [PubMed: 19878167]

23. Chen YW, Lai YC, Hsu CC, Chuang YW, Hou MF. Value of nodal drainage patterns and tumor location from lymphoscintigraphic mapping in detecting axillary sentinel lymph node status in breast cancer: experience at Kaohsiung Medical University Hospital. Kaohsiung. J Med Sci. 2005 Jun; 21(6):251-257.

24. Rimsten A. Symptoms and signs in benign and malignant tumours of the breast. Upsala J Med Sci. 1976; 81:54-60. [PubMed: 944960]

25. Alam I, Awad ZT, Given HF. Cystosarcoma phyllodes of the breast: a clinicopathological study of 11 cases. Ir Med J. 2003; 96:179-180. [PubMed: 12926760]

26. Harvey PW, Darbre P. Endocrine disrupters and human health: Could oestrogenic chemicals in body care cosmetics adversely affect breast cancer incidence in women? A review of evidence and call for further research. J Appl Toxicol. 2004; 24:167-176. [PubMed: 15211609]

27. McGrath KG. An earlier age of breast cancer diagnosis related to more frequent use of antiperspirants/deodorants and underarm shaving. Eur J Cancer Prevent. 2003; 12:479-485.

28. Ellsworth DL, Ellsworth RE, Love B, et al. Outer breast quadrants demonstrate increased levels of genomic instability. Ann Surg Oncol. 2004; 11:861-868. [PubMed: 15313734] 


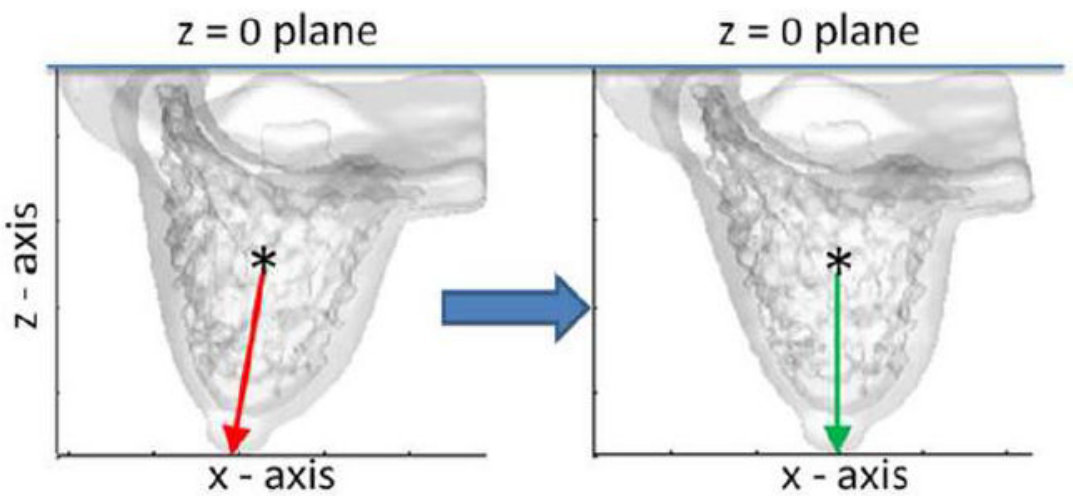

Figure 1.

Correction of the breast orientation based on the centroid-nipple line. 


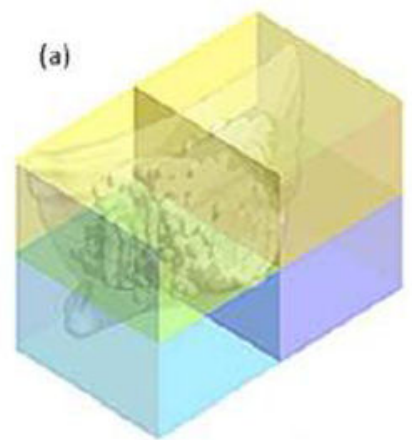

(b)
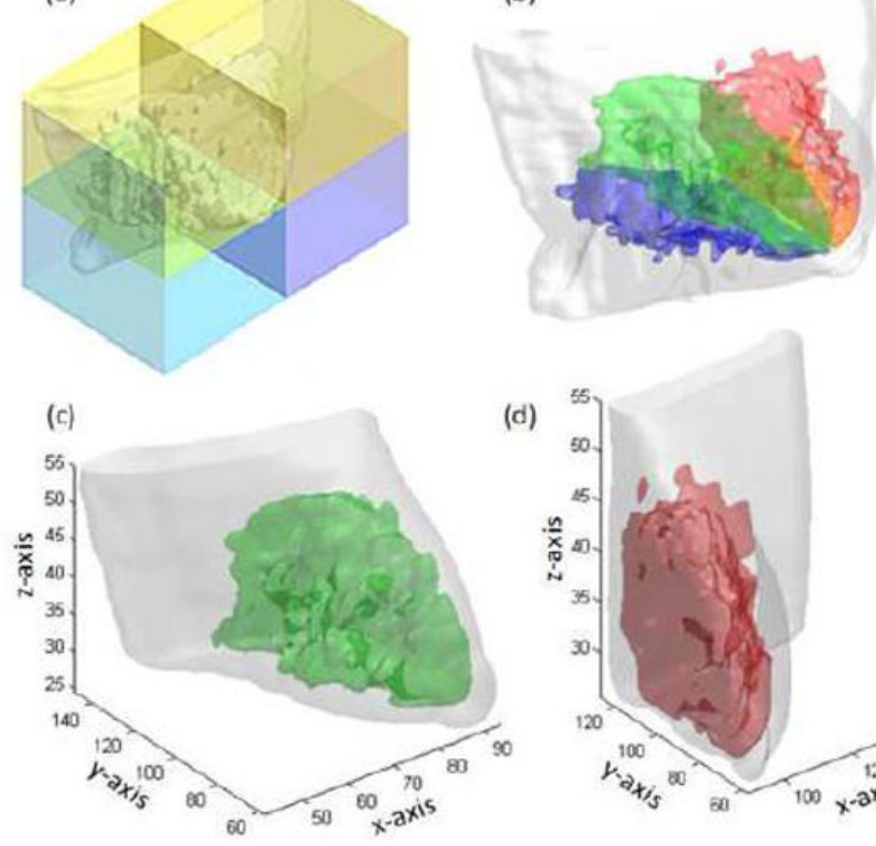

(d)

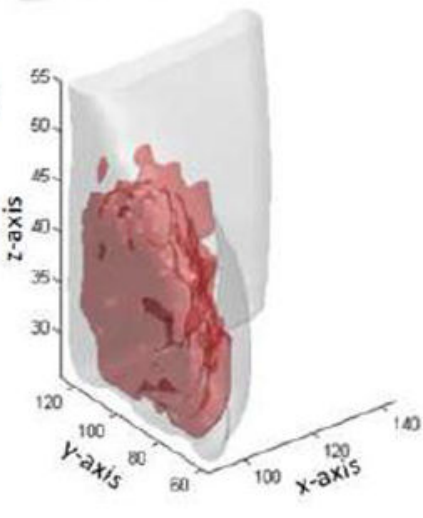

Figure 2.

Division of breast tissue and fibroglandular tissue into four quadrants, and the separated UO and UI quadrants. 


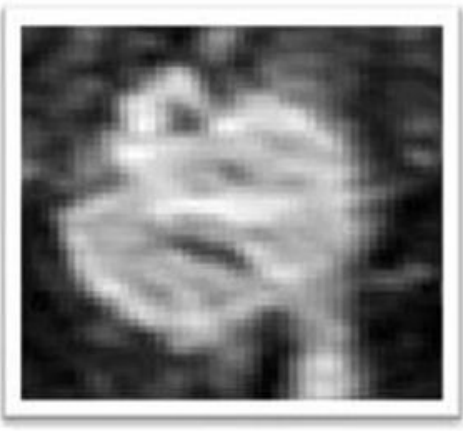

(a)

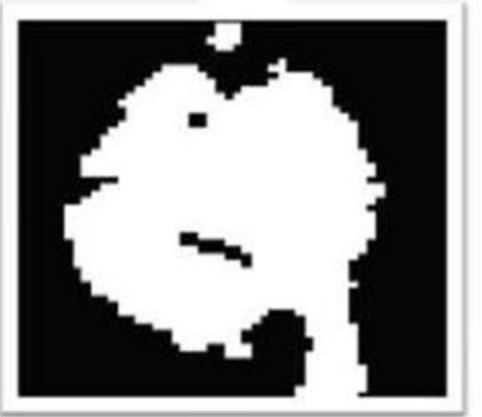

(c)

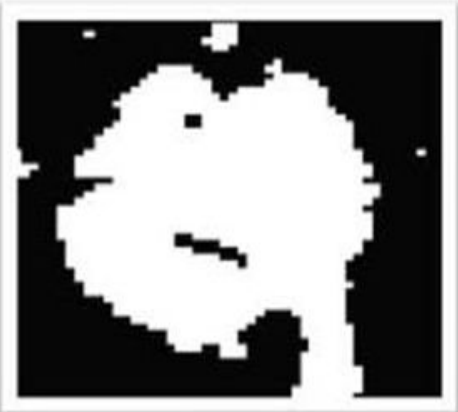

(b)

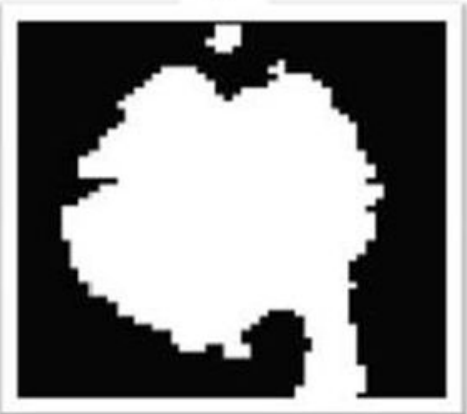

(d)

Figure 3.

Illustration of the tumor segmentation process on one image slice. (a) contrast enhancement map; (b) tumor voxels determined after FCM clustering segmentation; (c) tumor mask after removing scattered voxels; (d) tumor mask after hole-filling. 

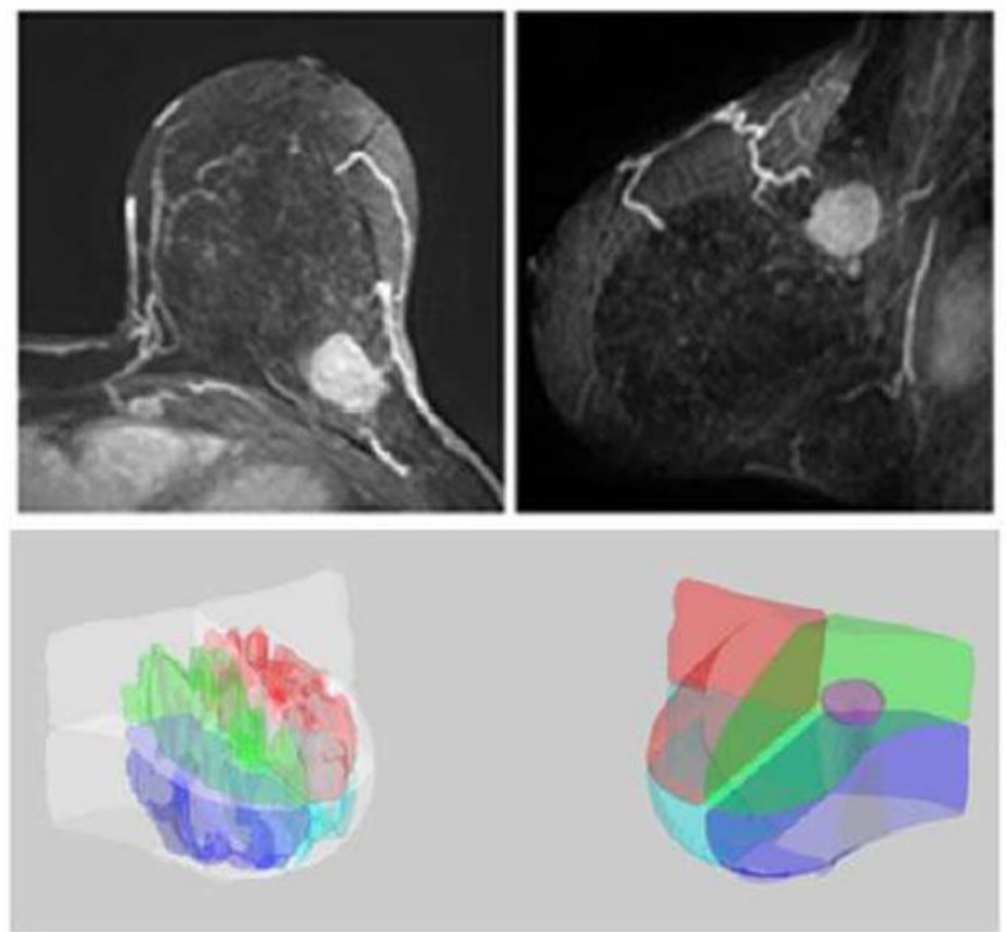

Figure 4.

A $65 \mathrm{y} / \mathrm{o}$ woman with a $2.5 \mathrm{~cm}$ invasive cancer in the UO quadrant of the left breast. The quadrant density in the right normal breast is the highest in UO quadrant (16.7\%) and the lowest in the LI quadrant (9.3\%). 

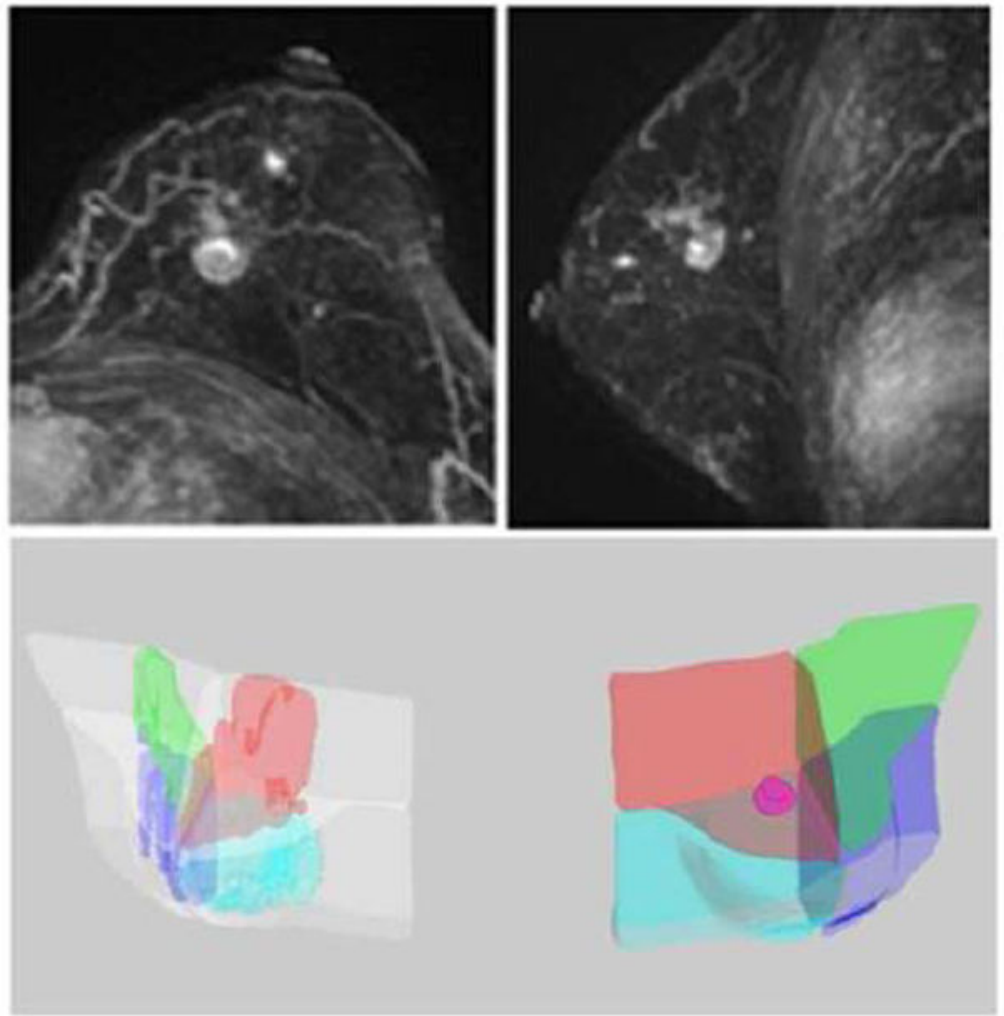

Figure 5.

A $56 \mathrm{y} / \mathrm{o}$ woman with a $1.3 \mathrm{~cm}$ invasive cancer in the UI quadrant of the left breast. The quadrant density in the right normal breast is highest in the UI quadrant (12.0\%) and the lowest in the LO quadrant (1.4\%). 

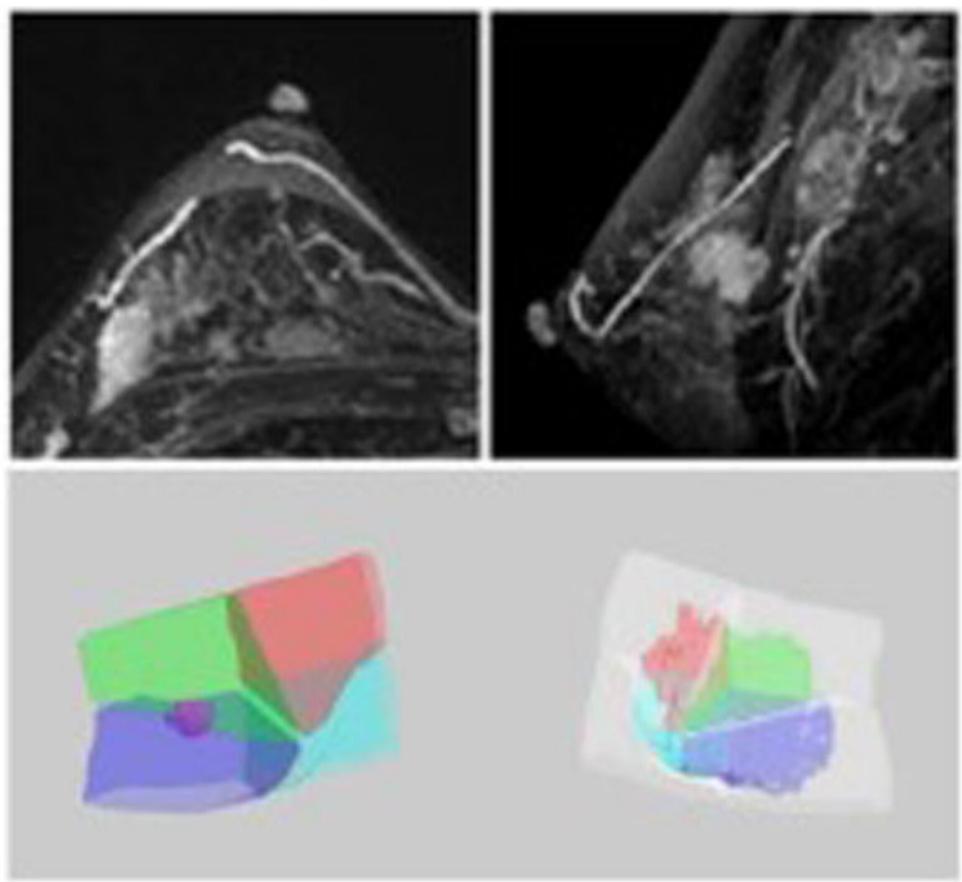

Figure 6.

A 38 y/o woman with a $2.9 \mathrm{~cm}$ invasive cancer predominantly in the LO quadrant of the right breast. The LO quadrant contained $69.1 \%$ of the total tumor volume; while the remaining tumor $(30.9 \%)$ was in the UO quadrant. The quadrant breast density in the left normal breast was highest in the UI quadrant $(21.9 \%)$ and lowest in the LI and LO quadrant (7.2\% and $7.2 \%$ ). The quadrant breast density in the UO quadrant was $14.0 \%$. 


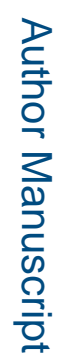

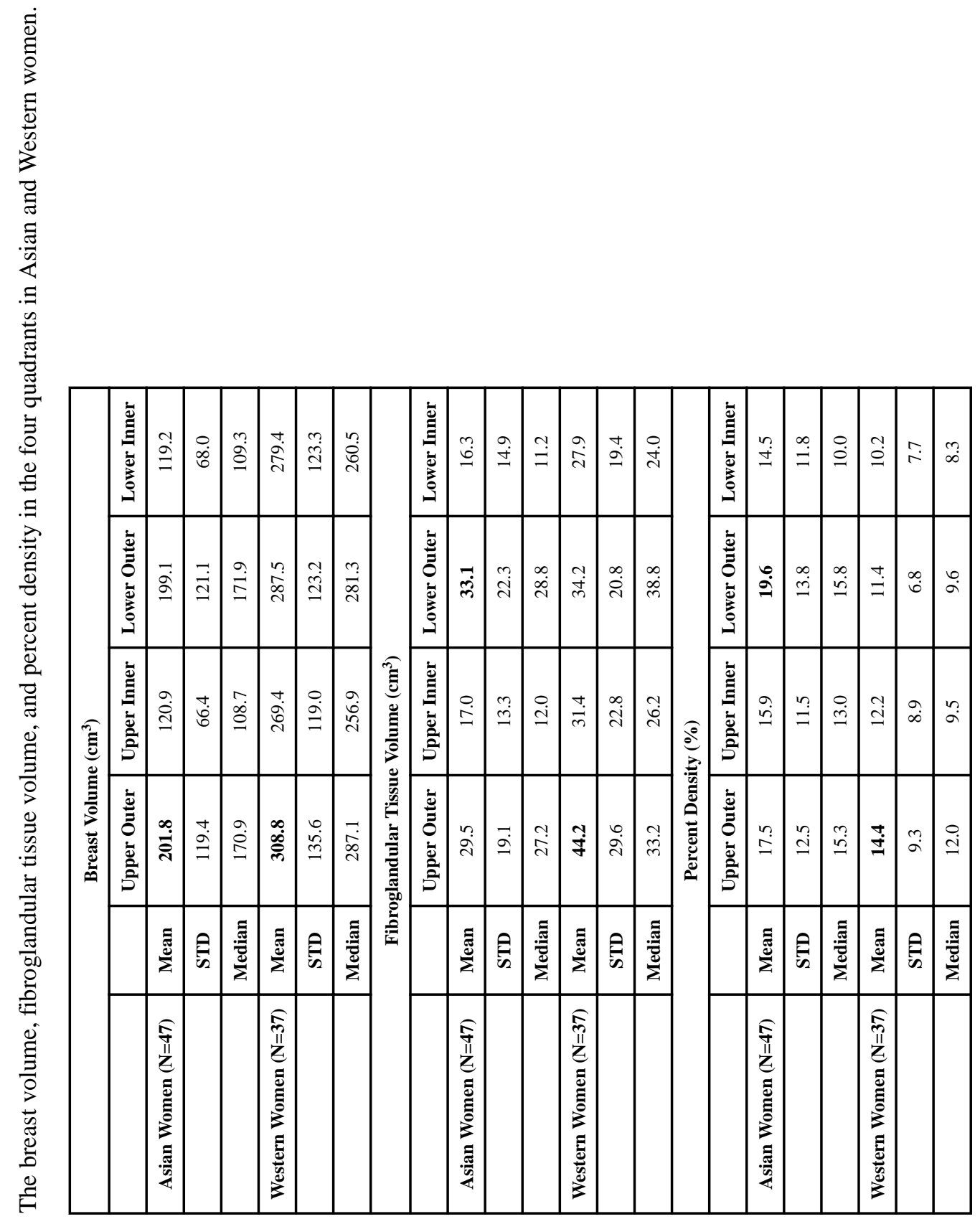

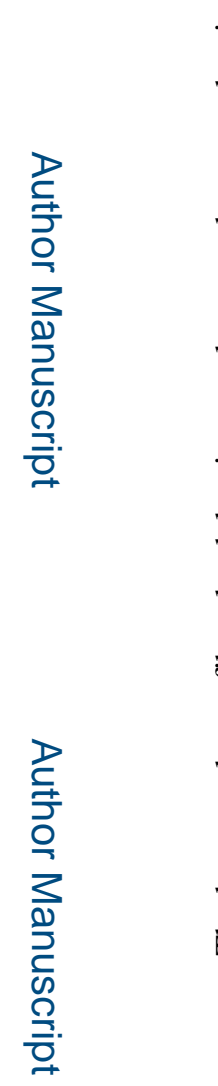



을

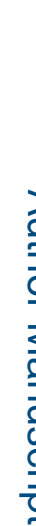

Acad Radiol. Author manuscript; available in PMC 2018 July 01 
Table 2

The distribution of breast cancer in the different quadrants of the breast

\begin{tabular}{|c|c|c|c|c|}
\hline & Upper Outer & Upper Inner & Lower Outer & Lower Inner \\
\hline All Subjects (N=84) & $36(42.9 \%)$ & $20(23.8 \%)$ & $17(20.2 \%)$ & $11(13.1 \%)$ \\
\hline Asian Women $(\mathbf{N}=\mathbf{4 7})$ & $21(44.7 \%)$ & $9(19.1 \%)$ & $7(14.9 \%)$ & $10(21.3 \%)$ \\
\hline Western Women $(\mathbf{N}=\mathbf{3 7})$ & $15(40.6 \%)$ & $11(29.7 \%)$ & $10(27.0 \%)$ & $1(2.7 \%)$ \\
\hline
\end{tabular}


Table 3

The distribution of highest QPD, and lesions occurring in the highest QPD quadrant

\begin{tabular}{|c|c|c|c|c|}
\hline & Upper Outer & Upper Inner & Lower Outer & Lower Inner \\
\hline \multicolumn{5}{|c|}{ Asian Women (N=47) } \\
\hline Highest QPD & $11(23.4 \%)$ & $8(17.0 \%)$ & $19(40.4 \%)$ & $9(19.2 \%)$ \\
\hline Lesions in Highest QPD & $5(45.5 \%)$ & $0(0 \%)$ & $2(10.5 \%)$ & $1(11.1 \%)$ \\
\hline \multicolumn{5}{|c|}{ Western Women (N=37) } \\
\hline Highest QPD & $20(54.1 \%)$ & $7(18.9 \%)$ & $8(21.6 \%)$ & $2(5.4 \%)$ \\
\hline Lesions in Highest QPD & $6(30.0 \%)$ & $2(28.6 \%)$ & $1(12.5 \%)$ & $0(0 \%)$ \\
\hline
\end{tabular}

